\title{
TWO TOPOLOGICAL PROBLEMS CONCERNING INFINITE-DIMENSIONAL NORMED LINEAR SPACES $\left({ }^{1}\right)$
}

\author{
BY \\ BOR-LUH LIN
}

Introduction. In the present work, we shall study the following two closely related topological problems concerning infinite-dimensional normed linear spaces.

Problem I. Given a closed subset $K$ of an infinite-dimensional normed linear space $E$, when is $E \sim K$ homeomorphic to $E$ ?

Problem II. Given a closed subset $K$ of an infinite-dimensional normed linear space $E$, when does there exist a periodic homeomorphism of $E$ onto $E$ with $K$ as its set of fixed points?

Using the fact that every nonreflexive Banach space contains a decreasing sequence of nonempty bounded closed convex subsets with empty intersection, Klee [11] proved that if $K$ is a compact subset of a nonreflexive Banach space $E$, then $E$ is homeomorphic to $E \sim K$. Later [13], he showed that every infinite-dimensional normed linear space contains a decreasing sequence of unbounded but linear bounded (for the definition, see \$1) nonempty closed convex subsets with empty intersection. He used this result to prove that every infinite-dimensional normed linear space $E$ is homeomorphic to $E \sim K$ where $K$ is an arbitrary compact subset of $E$. As a consequence [13], if $C$ is the unit cell of an infinite-dimensional normed linear space $E$, then there exists a homeomorphism $i$ of $C$ onto a closed half-space $J$ in $E$ such that $i(\operatorname{Bd} C)=\operatorname{Bd} J$. Klee [11] also proved that if $E$ is either a nonreflexive strictly convexifiable Banach space or an infinite-dimensional $l_{p}$-space, then $Q$ is homeomorphic to $Q \cup K$ where $K$ is a compact convex subset of the bounding hyperplane of an open halfspace $Q$ in $E$.

Concerning the set of fixed points of a periodic homeomorphism of a topological space into itself, a classical result of Smith [19] states that if $M$ is a finite-dimensional locally compact space, acyclic $\bmod p$ where $p$ is

Presented to the Society, May 6,1963, under the titles On a theorem of Klee and Some topological properties of infinite-dimensional Banach spaces; received by the editors August $19,1963$.

(') This work was partially supported by National Science Foundation Grant G24865 and represents the major part of a doctoral thesis submitted to Northwestern University in August, 1963. The author wishes to express his thanks to Professor Ky Fan for his many helpful suggestions. 
a prime, then the set of fixed points of every homeomorphism of period $p$ of $M$ into $M$ is acyclic $\bmod p$. For infinite-dimensional Hilbert space, the situation is quite different. A result of Klee [14] asserts that if $K$ is a compact subset of an infinite-dimensional Hilbert space $E$, then for each integer $n>1$, there exists a homeomorphism of period $n$ of $E$ onto $E$ with $K$ as its set of fixed points.

We shall continue the study in these directions and strengthen some of the known results. We first prove that in every infinite-dimensional normed linear space there exists a sequence $\left\{C_{n}\right\}$ of unbounded but linearly bounded closed convex bodies with empty intersection and Int. $C_{n} \supset C_{n+1}$ for each $n=0,1,2, \ldots$. Then we are able to prove (Theorem 1.3) that if $K$ is a bounded closed convex subset of an infinite-dimensional normed linear space $E$, then there exists a homeomorphism $i$ of $E \times[0,1]$ onto $(E \times(0,1])$ $\cup((E \sim K) \times\{0\})$ such that $i(E \times\{0\})=(E \sim K) \times\{0\}$ and $i(x, 1)$ $=(x, 1)$ for all $x$ in $E$. Applying a result of Bartle and Graves [2] and Theorem 1.3, we shall show in Theorem 3.5 that for every closed subset $K$ of a closed linear subspace $F$ of infinite deficiency in a Banach space $E$, $E \sim K$ is homeomorphic to $E$. If, in addition, $Q$ is an open half-space in $E$ such that the bounding hyperplane of $Q$ contains $F$, then $Q$ is homeomorphic to $Q \cup K$. Using these properties of an infinite-dimensional Banach space $E$, we can show (Theorem 5.3) that for every closed subset $K$ of a closed linear subspace of infinite deficiency in $E$ and for each positive integer $n$, there exists a homeomorphism of period $2 n$ of $E$ onto $E$ with $K$ as its set of fixed points; in case $E$ is a Hilbert space, then for each integer $n>1$, there exists a homeomorphism of period $n$ of $E$ onto $E$ with $K$ as its set of fixed points. Now, for every compact subset $K$ of an infinite-dimensional Hilbert space $E$, there exists a homeomorphism $i$ of $E$ onto $E$ such that $i(K)$ is contained in a closed linear subspace of infinite deficiency in $E$ [12], hence Klee's result concerning the set of fixed points of a periodic homeomorphism, mentioned in the last paragraph, is a consequence of Theorem 5.3.

The above results concerning Problems I, II deal with those sets $K$ which are "small" in the sense that $K$ is a closed subset of a closed linear subspace of infinite deficiency in a Banach space. Next, we shall consider those sets $K$ which are "large" in the sense that $K$ is a closed convex body of an infinite-dimensional closed linear subspace of a Banach space $E$. For a closed convex body $K$ in an infinite-dimensional Banach space $E$, the characteristic cone $\{y \in E \mid x+[0, \infty)(y-x) \subset K\}$ of $K$ relative to $x \in K$ is either a linear variety of finite deficiency, a linear variety of infinite deficiency, or not a linear variety. We shall show in Theorems 2.5, 2.6 that, if the characteristic cone of a closed convex body $K$ of an infinitedimensional Banach space $E$ is not a linear variety of finite deficiency in 
$E$, then there exists a homeomorphism $i$ of $E$ onto $E$ such that $i(K)$ is a closed half-space $J$ in $E$ and $i(\operatorname{Bd} K)$ is the bounding hyperplane of $J$. Hence, by using Theorem 1.3 , we can prove (Theorem 3.1 ) that if $F$ is an infinite-dimensional closed linear subspace of a Banach space $E$ and $K$ is a closed convex body in $F$ such that the characteristic cone of $K$ is not a linear variety of finite deficiency in $E$, then $E$ is homeomorphic to $E \sim K$. In case the characteristic cone of $K$ is a linear variety of finite deficiency, $E$ is not necessarily homeomorphic to $E \sim K$. For example, $E$ is not homeomorphic to $E \sim H$, where $H$ is a closed hyperplane in $E$. On the other hand, if $K$ is a closed convex body on the bounding hyperplane of an open half-space $Q$ of an infinite-dimensional Banach space $E$, it is unknown whether or not $Q$ and $Q \cup K$ are homeomorphic. In particular, it is unknown whether or not $Q$ is homeomorphic to $Q \cup \operatorname{Bd} Q$. Since $Q \cup \operatorname{Bd} Q$ is homeomorphic to the unit cell of $E$ (Theorem 2.4), this is equivalent to the following problem: When is an infinite-dimensional Banach space homeomorphic to its unit cell? This leads to the following question: Let $K$ be a closed convex body of a closed linear subspace of finite deficiency in an infinite-dimensional Banach space $E$. When is $K$ homeomorphic to $E$ ? Klee [11] showed that every infinite-dimensional Hilbert space is homeomorphic to its unit cell and unit sphere. Later [16], he showed that if a Banach space $E$ contains an $h$-compressible proper closed linear subspace, then $E$ is homeomorphic to its unit cell. In Theorem 4.3, we show that if a Banach space $E$ contains a closed linear subspace $B$ of infinite deficiency such that $B$ is homeomorphic to $l_{2}$, if $K$ is a closed convex body of a closed linear subspace $F$ of finite deficiency in $E$, then $K$ is homeomorphic to $E$ and $\operatorname{Bd}_{F} K$ is homeomorphic either to $E$ or $E \times S_{n}$ for some non-negative integer $n$, where $S_{n}$ is the $n$-sphere. Concerning Problem II, Theorem 5.2 states that if $F$ is an infinite-dimensional closed linear subspace of a Banach space $E$ and $K$ is a closed convex body in $F$ such that the characteristic cone of $K$ is not a linear variety of finite deficiency in $F$, then, for each positive integer $n$, there exists a homeomorphism of period $2 n$ of $E$ onto $E$ with $\mathrm{Bd}_{F} K$ as its set of fixed points; in case $E$ is an infinite-dimensional Hilbert space, then, for each integer $n>1$, there exists a homeomorphism of period $n$ of $E$ onto $E$ with $K$ as its set of fixed points.

Notations. All topological vector spaces considered are real vector spaces with the Hausdorff property. The field of real numbers is denoted by $R,[m, n]=\{\alpha \in R \mid m \leqq \alpha \leqq n\}, \quad(m, n]=\{\alpha \in R \mid m<\alpha \leqq n\}, \quad$ and $\quad(m, n)$ $=\{\alpha \in R \mid m<\alpha<n\}$. For $x, y$ in a vector space $E$,

$$
(x, y]=\{\alpha x+(1-\alpha) y \mid 0 \leqq \alpha<1\} .
$$

The empty set is denoted by $\emptyset . E \sim A=\{x \in E \mid x \notin A\}$. For a subset $K$ of a subspace $F$ of a topological space $E$, Int. $K$ will denote the interior of 
$K$ in $E, \bar{K}$ will denote the closure of $K$ in $E$ and $\operatorname{Bd}_{F} K$ will denote the boundary of $K$ relative to $F$. For two topological spaces $E, F, E \approx F$ will mean that $E$ is homeomorphic to $F$.

1. Preliminary theorems. A closed convex subset $K$ of an Euclidean space is bounded if and only if its intersection with each line is bounded. This is not true in an infinite-dimensional normed linear space. The following terminology is introduced by Klee [12].

A subset of a linear space is said to be linearly bounded if its intersection with each line is a bounded subset.

Theorem 1.1. In every infinite-dimensional normed linear space $E$, there exists a sequence $\left\{C_{n}\right\}$ of unbounded but linearly bounded closed convex bodies such that $\bigcap_{n=0}^{\infty} C_{n}=\emptyset$ and Int. $C_{n} \supset C_{n+1}, C_{n} \not \equiv \emptyset$, for each $n=0,1,2, \cdots$.

Proof. Case 1. $E$ is separable.

By a theorem of Klee [13], there exists a sequence of continuous linear functionals $\left\{f_{i}\right\}, \bigcap_{i=1}^{\infty} f_{i}^{-1}(0)=\{0\},\left\|f_{i}\right\|=1$ for each $i=1,2, \cdots$ and a sequence of positive integers $\left\{n_{i}\right\}$ such that the closed convex set $D_{0}$ $=\bigcap_{i=1}^{\infty} f_{i}^{-1}\left(\left[-n_{i}, n_{i}\right]\right)$ is an unbounded but linearly bounded subset of $E$. By the uniform boundedness principle [6], $E$ admits a continuous linear functional $f,\|f\|=1$, such that $\sup _{D_{0}} f=\infty$. Let $D_{n}=D_{0} \cap f^{-1}[n, \infty)$ for $n=1,2, \cdots$. Then $\left\{D_{n}\right\}$ is a decreasing sequence of unbounded but linearly bounded closed convex sets with empty intersection.

For a given $\epsilon>0$ and a subset $A$ of $E$, let $N_{\epsilon} A=\{x \in E \mid\|x-a\|<\epsilon$ for some $a$ in $A\}$. It is clear that if $A$ is a convex subset of $E$, then $N_{\epsilon} A$ is also a convex subset.

We claim that for any $\epsilon, 0<\epsilon<\infty, N_{\epsilon} D_{n}$ is a linearly bounded subset of $E$ for each $n=0,1,2, \cdots$.

For an arbitrary $x$ in $E$, there is an integer $i$ such that $f_{i}(x)=\delta \neq 0$. Choose $m$ so that $m|\delta|>n_{i}+\epsilon$. Then for any $\alpha,|\alpha| \geqq m$, and any $y \in f_{i}^{-1}\left(\left[-n_{i}, n_{i}\right]\right) \quad$ we have $\epsilon<m|\delta|-n_{i} \leqq|\alpha||\delta|-n_{i} \leqq|\alpha \delta|-\left|f_{i}(y)\right|$ $\leqq\left|f_{i}(\alpha x-y)\right| \leqq\left\|f_{i}\right\| \cdot\|\alpha x-y\|=\|\alpha x-y\|$. Since $D_{n} \subset f_{i}^{-1}\left(\left[-n_{i}, n_{i}\right]\right)$, this implies that $\alpha x$ is not in $N_{\epsilon} D_{n}$ for all $\alpha,|\alpha| \geqq m$. Since $N_{\epsilon} D_{n}$ is convex, it follows easily that $N_{\epsilon} D_{n}$ is a linearly bounded subset for each $n=0,1,2, \cdots$.

Let $C_{n}=\overline{N_{1 / 2^{n}} D_{n}}, n=0,1,2, \ldots$. It is easy to prove that $C_{n} \subset N_{1 / 2^{n-1}} D_{n}$. We have shown that $N_{\epsilon} D_{n}$ is linearly bounded for each $n=0,1,2, \cdots$ and each $\epsilon>0$. Hence $\left\{C_{n}\right\}$ is a sequence of unbounded but linearly bounded closed convex bodies, Int. $C_{n} \supset C_{n+1}$ for each $n=0,1,2, \cdots$. It remains to show that $\bigcap_{n=0}^{\infty} C_{n}=\emptyset$.

Suppose $x \in \bigcap_{n=0}^{\infty} C_{n}$. For each integer $m \geqq 0$, there exist $y_{m n} \in N_{1 / 2^{n}} D_{n}$ such that $\left\|x-y_{m n}\right\|<1 / 2^{m}$ for each $n=0,1,2, \ldots . y_{m n} \in N_{1 / 2^{n}} D_{n}$ implies that there exists $z_{m n} \in D_{n}$ such that $\left\|y_{m n}-z_{m n}\right\|<1 / 2^{n}$. Hence $\left\|x-z_{n n}\right\|$ 
$\leqq\left\|x-y_{n n}\right\|+\left\|y_{n n}-z_{n n}\right\|<1 / 2^{n}+1 / 2^{n}=1 / 2^{n-1} \rightarrow 0$ as $n \rightarrow \infty$. Since $\left\{D_{n}\right\}$ is a decreasing sequence of closed subsets and $z_{n n} \in D_{n}$ for each $n=0,1,2, \cdots$, this implies that $x \in D_{n}$ for each $n=0,1,2, \cdots$, contradiction.

Case 2. $E$ is not separable.

Let $F$ be a separable closed linear subspace of $E$. By Case 1, there exists a sequence of unbounded but linearly bounded closed convex subsets $\left\{D_{n}\right\}$ of $F$ with empty intersection. For each $x \in E \sim F$, by the Hahn-Banach theorem, there is a continuous linear functional $g$ on $E$ such that $g(F)$ $=0, g(x)=\delta \neq 0$. By an argument similiar to the argument of Case 1, it can be shown that for each $\epsilon, 0 \leqq \epsilon<\infty, N_{\epsilon} D_{n}=\{x \in E \mid\|x-a\|<\epsilon$ for some $a$ in $\left.D_{n}\right\}$ is a linearly bounded subset of $E$, for each $n=0,1,2, \ldots$. Let $C_{n}=\overline{N_{1 / 2}{ }^{n} D_{n}}$, then $\left\{C_{n}\right\}$ is a sequence of unbounded but linearly bounded closed convex bodies in $E$ with empty intersection and Int. $C_{n}$ $\supset C_{n+1}$ for each $n=0,1,2, \cdots$ Q.E.D.

The following concept introduced by Steinitz and also studied by Stoker $[20]$ is useful in classifying the closed convex subsets in a topological vector space (see §2): Let $A$ be a closed convex subset of a topological vector space $E$ and $x \in A$. The characteristic cone of $A$ relative to $x$ is the $\operatorname{set} \operatorname{cc}(A ; x)$ $=\{y \in E \mid x+[0, \infty)(y-x) \subset A\}$. It is clear that if $x, y \in A$, then $\operatorname{cc}(A ; x)$ $=\operatorname{cc}(A ; y)+(x-y)$. Thus if no confusion is possible, we shall simply speak of the characteristic cone of $A$.

We need the following lemma of Corson and Klee [4] in proving Theorem 1.3.

Lemma 1.2. Suppose $E_{i}, i=1,2$, are topological vector spaces, $A_{i}, B_{i}$ are closed convex bodies in $E_{i}$ and $y_{i} \in E_{i}$ such that $y_{i} \in \operatorname{Int} . A_{i} \subset A_{i} \subset$ Int. $B_{i}$ and $\operatorname{cc}\left(A_{i} ; y_{i}\right)=\operatorname{cc}\left(B_{i} ; y_{i}\right)$. Then every homeomorphism $h$ of $\operatorname{Bd} B_{1}$ onto $\mathrm{Bd} B_{2}$ can be extended to a homeomorphism $k$ of $B_{1} \sim$ Int. $A_{1}$ onto $B_{2} \sim \operatorname{Int} . A_{2}$ such that $k(x) \in\left(y_{2}, h(z)\right]$ whenever $z \in \operatorname{Bd} B_{1}$ and $x \in\left(y_{1}, z\right] \sim$ Int. $A_{1}$.

Theorem 1.3. For every bounded closed convex subset $K$ of an infinitedimensional normed linear space $E$, there exists a homeomorphism $i$ of $E \times[0,1]$ onto $(E \times(0,1]) \cup((E \sim K) \times\{0\})$ such that $i(x, 1)=(x, 1)$ for all $x$ in $E$ and $i(E \times\{0\})=(E \sim K) \times\{0\}$.

Proof. By Theorem 1.1, there exists a sequence $\left\{C_{n}\right\}$ of unbounded but linearly bounded closed convex bodies such that Int. $C_{n} \supset C_{n+1}$ for each $n=0,1,2, \cdots$ and $\bigcap_{n=0}^{\infty} C_{n}=\emptyset$. By considering $\left\{\alpha C_{n}\right\}$, for some $\alpha \in R$, instead of $\left\{C_{n}\right\}$, if necessary, we may assume that $K \subset$ Int. $C_{0}$.

Let $F=E \times R$ be the product space of $E$ and $R$. Define

$$
A_{n}=C_{n} \times\left[-\frac{1}{n+1}, \frac{1}{n+1}\right], \quad n=0,1,2, \cdots .
$$


Choose $y_{n} \in$ Int. $C_{n}, n=0,1,2, \ldots$. Then the sequence $\left\{A_{n}\right\}$ has the following properties:

$A_{n}$ is a closed convex body in $F,\left(y_{n}, 0\right) \in \operatorname{Int} . A_{n}$ and Int. $A_{n} \supset A_{n+1}$ for all $n=0,1,2, \cdots \cdot \operatorname{cc}\left(A_{i},\left(y_{n}, 0\right)\right)=\left(y_{n}, 0\right)$ for each $0 \leqq i \leqq n . \bigcap_{n=0}^{\infty} A_{n}=\emptyset$.

Now, let $B_{0}=A_{0}$. Let $\epsilon$ be a positive number such that $B_{0}$ contains the $2 \epsilon$-neighborhood $N_{2 \epsilon}(K \times\{0\})$ of $K \times\{0\}$. For $n \geqq 1$, let $B_{n}$ $=\overline{N_{\epsilon / n}(K \times\{0\})}$. Let $z_{n}=(0,0)$ for all $n=0,1,2, \ldots$. Then the sequence $\left\{B_{n}\right\}$ has the following properties:

$B_{n}$ is a closed convex body in $F, z_{n} \in \operatorname{Int} . B_{n}$, Int. $B_{n} \supset B_{n+1}$ for all $n=0,1,2, \cdots \cdot \operatorname{cc}\left(B_{i}, z_{n}\right)=z_{n}$ for all $0 \leqq i \leqq n$ and $\bigcap_{n=0}^{\infty} B_{n}=K \times\{0\}$.

Let $i_{0}$ be the identity mapping of $F \sim \operatorname{Int} . A_{0}$. Then $i_{0}\left(\operatorname{Bd} A_{0}\right)=\operatorname{Bd} B_{0}$. By Lemma 1.2, $i_{0} \mid \mathrm{Bd} A_{0}$ can be extended to a homeomorphism $i_{1}$ of $A_{0}$ $\sim$ Int. $A_{1}$ onto $B_{0} \sim$ Int. $B_{1}$ which takes points of $E \times\{0\}$ into $E \times\{0\}$. Continuing in this way, we obtain a sequence of homeomorphisms $i_{0}, i_{1}, i_{2}, \cdots$ such that $i_{n}\left(A_{n-1} \sim \operatorname{Int} . A_{n}\right)=B_{n-1} \sim \operatorname{Int} . B_{n}$ for each $n=1$, $2,3, \cdots$ Let $i=\bigcup_{n=0}^{\infty} i_{n}$. Since $\bigcap_{n=0}^{\infty} A_{n}=\emptyset, \quad \bigcap_{n=0}^{\infty} B_{n}=K \times\{0\}$, it follows that $i$ is a homeomorphism of $F$ onto $F \sim(K \times\{0\})$. The restriction of $i$ on $E \times[0,1]$ is a homeomorphism of $E \times[0,1]$ onto $(E \times(0,1])$ $\cup((E \sim K) \times\{0\})$ such that $i(x, 1)=(x, 1)$ for all $x$ in $E$ and $i(E \times\{0\})$ $=(E \sim K) \times\{0\}$. Q.E.D.

2. Classification of closed convex bodies. We begin with a result of Bartle and Graves [2], extended to the present form (Lemma 2.1) by Michael [18]. A consequence (Corollary 2.3) of the result is needed in the proofs of Theorem 2.5 and most of the results in $\$ 3,4,5$.

Lemma 2.1 (BARTle-Graves). Let $f$ be a continuous linear mapping of a Banach space $E$ onto a Banach space $F$. Then there exist a positive constant $m$ and a continuous mapping $g$ of $F$ into $E$ such that $f \circ g(x)=x, g(\alpha x)$ $=\alpha g(x),\|g(x)\| \leqq m\|x\|$ for all $x$ in $F$ and $\alpha$ in $R$.

Let $G=f^{-1}(0)$. For each $(x, y) \in F \times G$, define

$$
\|(x, y)\|=\max (\|x\|,\|y\|) \text {. }
$$

It can be proved that $F \times G$ is a Banach, space with the norm \|\| .

TheOREM 2.2. Let $f$ be a continuous linear mapping of a Banach space $E$ onto a Banach space $F$. Then there exists a homeomorphism $h$ of $E$ onto $F \times G$, $G=f^{-1}(0)$, such that $h(y)=(0, y)$ for all $y$ in $G$ and $\|h(y)\|=\|y\|$ for all $y \in E$.

Proof. By the previous lemma, there is a continuous mapping $g$ of $F$ into $E$ such that $f \circ g(x)=x, g(\alpha x)=\alpha g(x),\|g(x)\| \leqq m\|x\|$ for all $x$ in $F$ and all $\alpha$ in $R$. Define $h_{1}: E \rightarrow F \times G$ by $h_{1}(y)=(f(y), y-g \circ f(y))$ for all $y$ in $E$. It can be proved that $h_{1}$ is a homeomorphism of $E$ onto $F \times G$ 
such that $h_{1}(y)=(0, y)$ for all $y$ in $E$. Define $h: E \rightarrow F \times G$ by $h(y)$ $=\|y\| h_{1}(y) /\left\|h_{1}(y)\right\|$ if $h_{1}(y) \neq 0$, that is, $y \neq 0$, and $h(0)=(0,0)$. Then $\|y\| /(1+m) \leqq\left\|h_{1}(y)\right\| \leqq((m+1)\|f\|+1)\|y\|$ for all $y$ in $E$ and $h$ is a homeomorphism of $E$ onto $F \times G$ such that $h(y)=(0, y)$ for all $y$ in $F$ and $\|h(y)\|=\|y\|$ for all $y$ in $E$. Q.E.D.

CoRollary 2.3. For any closed linear subspace $F$ of a Banach space $E$, there exists a homeomorphism $h$ of $E$ onto $(E / F) \times F$ such that $h(y)=(0, y)$ for all $y$ in $F$.

Proof. Let $f$ be the canonical mapping of $E$ onto the Banach space $E / F$. The corollary follows immediately from Theorem 2.2. Q.E.D.

Clearly, the characteristic cone of a closed convex body in an infinitedimensional normed linear space is either a linear variety of infinite deficiency or a linear variety of finite deficiency or is not a linear variety. We shall consider each case separately.

Theorem 2.4. Let $E$ be an infinite-dimensional normed linear space, $C=\{x \in E \mid\|x\| \leqq 1\}$ and $S=\{x \in E \mid\|x\|=1\}$. Let $J$ be a closed halfspace in $E$ with bounding hyperplane $H$. Then there exists a homeomorphism $i$ of $E$ onto $E$ such that $i(C)=J$ and $i(S)=H$.

Proof. By Theorem 1.3, $E$ is homeomorphic to $E \sim\{0\}$. Using this property of $E$, Klee [13] proved that there exists a homeomorphism $j$ of $C$ onto $J$ which maps $S$ onto $H$. Without loss of generality, we may assume $0 \in J \sim H$. Define $i$ of $E$ onto $E$ by $i(x)=j(x)$ if $x \in C, i(x)=\|x\| j(x /\|x\|)$ if $x \notin C$. It is clear that $i$ is a homeomorphism and $i(C)=J, i(S)=H$. Q.E.D.

Theorem 2.5. Let $K$ be a closed convex body in a Banach space $E$. If the characteristic cone of $K$ is a closed linear variety $L$ of infinite deficiency in $E$, then there exists a homeomorphism $i$ of $E$ onto $E$ such that $i(K)$ is a closed half-space $J$ in $E$ and $i(\operatorname{Bd} K)$ is the bounding hyperplane of $J$.

Proof. We may assume that $0 \in$ Int. $K, 0 \in L$.

Case 1. Suppose $L=\{0\}$, that is, $K$ is a linearly bounded closed convex body in $E$.

For any point $y \neq 0$ in $E$, there exists a unique point $x$ in $\operatorname{Bd} K$ such that $y=\alpha x$ for some scalar $\alpha>0$. Define $j: E \rightarrow E$ by

$$
j(y)=\alpha \frac{x}{\|x\|} \quad \text { for all } y \neq 0 \text { in } E ; \quad y=\alpha x, x \in \operatorname{Bd} K,
$$

and

$$
j(0)=0 .
$$


Then $j$ is a homeomorphism of $E$ onto $E$ such that $j(K)=C$ and $j(\operatorname{Bd} K)$ $=S$. Let $i_{1}$ be a homeomorphism of $E$ onto $E$ such that $i_{1}(C)$ is a closed half-space $J$ and $i_{1}(S)$ is the bounding hyperplane $H$ of $J$ obtained in Theorem 2.4. Then $i=i_{1} \circ j$ is a homeomorphism of $E$ onto $E, i(K)=J$, $i(\operatorname{Bd} K)=H$.

Case 2. Suppose $\operatorname{dim} L \geqq 1$.

We claim that $K=\bigcup_{x \in K}(x+L)$.

It is clear that $K \subset \bigcup_{x \in K}(x+L)$. Given any $x \in K, y \in L$, since $y / \lambda$ $\in L$ for all real numbers $\lambda>0$ and $K$ is convex, $(1-\lambda) x+\lambda(y / \lambda)$ $=(1-\lambda) x+y$ is in $K$ for all $0<\lambda \leqq 1$. Let $\lambda \rightarrow 0$; then we have $x+y$ $\in K$ because $K$ is closed.

By Corollary 2.3, the mapping $h$ of $E$ onto $(E / L) \times L$, defined by $h(x)$ $=(f(x), x-g \circ f(x))$ for all $x$ in $E$ where $f$ is the canonical mapping of $E$ onto $E / L$, is a homeomorphism. Since $K=\bigcup_{x \in K}(x+L)$ and the characteristic cone of $K$ is $L, f(K)$ is a linearly bounded closed convex body in $E / L$ and $h(K)=f(K) \times L$. Let $J$ be a closed half-space in $E$; we may assume that the bounding hyperplane $H$ of $J$ contains $L$. By Case 1, there exists a homeomorphism $i$ of $E / L$ onto $E / L$ such that $i(f(K))=J / L$, $i(\operatorname{Bd} f(K))=H / L$. The mapping $i \times 1, \quad(i \times 1)(x, y)=(i(x), y)$ for all $(x, y)$ in $(E / L) \times L$, is a homeomorphism of $(E / L) \times L$ onto $(E / L) \times L$ which maps $f(K) \times L$ onto $(J / L) \times L,(\operatorname{Bd} f(K)) \times L=\operatorname{Bd} h(K)$ onto $(H / L)$ $\times L$. Hence $j=h^{-1} \circ(i \times 1) \circ h$ is a homeomorphism of $E$ onto $E, j(K)$ $=J, j(\mathrm{Bd} K)=H$. Q.E.D.

REMARK. Using the argument similar to the proof of Theorem 1.3, Corson and Klee [4] proved that if $F$ is a closed linear subspace of infinite deficiency in a normed linear space $E$, then $E$ is homeomorphic to $E \sim F$. They were then able to prove Theorem 2.5 in case $E$ is an infinitedimensional normed linear space. However, the proof of Theorem 2.5 is simpler.

Theorem 2.6. Let $K$ be a closed convex body in a normed linear space $E$. If the characteristic cone of $K$ is not a linear variety then there is a homeomorphism $i$ of $E$ onto $E$ such that $i(K)$ is a closed half-space $J$ in $E$ and $i(\mathrm{Bd} K)$ is the bounding hyperplane of $J$.

Proof. We may assume $0 \in K, 0 \in J \sim H$. There is a homeomorphism $j$ of $K$ onto $J$ such that $j(\operatorname{Bd} K)=H, j(K)=J[11$, p. 30]. For each element $y$ in $E \sim K$, there exists a unique point $x$ in $\operatorname{Bd} K$ such that $y=\alpha x$ for some scalar $\alpha>0$. Define $i$ of $E$ onto $E$ by $i(y)=j(y)$ if $y$ is in $K$, $i(y)=\alpha j(x)$ if $y \in E \sim K, y=\alpha x, x \in \operatorname{Bd} K$. It can be proved that $i$ is a homeomorphism of $E$ onto $E, i(K)=J, i(\operatorname{Bd} K)=H$. Q.E.D.

The above two results are to be compared with the following result of Klee [11]. 
THEOREM 2.7. If the characteristic cone of a closed convex body $K$ of a normed linear space $E$ is a linear variety $L$ of finite deficiency $n$ in $E$, then there is a homeomorphism $i$ of $K$ onto $L \times C_{n}$ where $C_{n}$ is the unit cell in $n$ dimensional Euclidean space and $i(\operatorname{Bd} K)=L \times S_{n-1}$ where $S_{n-1}$ is the $(n-1)$-sphere.

3. Closed subsets $K$ of an infinite-dimensional normed linear space $E$ such that $E$ and $E \sim K$ are homeomorphic. First, we consider the case when $K$ is a closed convex body of an infinite-dimensional closed linear variety of a Banach space $E$. Then we shall consider the case when $K$ is a closed subset of a closed linear variety of infinite deficiency in a Banach space $E$.

TheOREM 3.1. Let $F$ be an infinite-dimensional closed linear variety of a Banach space $E$. If the characteristic cone of a closed convex body $K$ of $F$ is not a linear variety of finite deficiency in $F$, then $E$ and $E \sim K$ are homeomorphic.

Proof. We may assume that $F$ is a closed linear subspace. Corollary 2.3 asserts that there is a homeomorphism $h$ of $E$ onto $(E / F) \times F$ such that $h(x)=(0, x)$ for all $x$ in $F$ and $\|h(x)\|=\|x\|$ for all $x$ in $E$. By Theorems $2.5,2.6$, there exists a homeomorphism $k$ of $F$ onto $F$ such that $k(K)$ $=C_{F}$, the unit cell of $F$. The mapping $i=h^{-1} \circ(1 \times k) \circ h,(1 \times k)(x, y)$ $=(x, k(y))$ for all $(x, y)$ in $(E / F) \times F$, is a homeomorphism of $E$ onto $E$ such that $i(K)$ is a closed convex subset of the unit cell of $E$. By Theorem 1.3 , there exists a homeomorphism $j$ of $E$ onto $E \sim i(K)$. The mapping $i^{-1} \circ j \circ i$ is a homeomorphism of $E$ onto $E \sim K$. Q.E.D.

REMARK. If the characteristic cone of $K$ is a linear variety of finite deficiency, then $E$ is not necessarily homeomorphic to $E \sim K$. For example, $E$ is not homeomorphic to $E \sim H$ when $H$ is a closed hyperplane in $E$.

Lemma 3.2. For every infinite-dimensional normed linear space $E$, there exists a homeomorphism $j$ of $((E \sim\{0\}) \times(0,1]) \cup(E \times\{0\})$ onto $E \times[0,1]$ such that $j(x, 0)=(x, 0)$ for all $x$ in $E$.

Proof. By Theorem 1.3, there exists a homeomorphism $i$ of $E \times[0,1]$ onto $(E \times(0,1]) \cup((E \sim\{0\}) \times\{0\})$ such that $i(E \times\{0\})=(E \sim\{0\})$ $\times\{0\}$. By identifying $(E \sim\{0\}) \times\{0\}$ with $E \sim\{0\}$, there is a homeomorphism $i_{0}$ of $E$ onto $E \sim\{0\}$ defined by $i_{0}(x)=i(x, 0)$ for all $x$ in $E$. Define

$$
j:((E \sim\{0\}) \times[0,1]) \cup(\{0\} \times\{0\}) \rightarrow E \times[0,1]
$$

by

$$
j(x, t)= \begin{cases}i\left(i_{0}^{-1}(x), t\right) & \text { if } t \neq 0 \\ (x, t) & \text { if } t=0\end{cases}
$$


It can be proved that $j$ is a homeomorphism of $((E \sim\{0\}) \times[0,1])$ $\cup(\{0\} \times\{0\})$ onto $E \times[0,1]$ such that $j(x, 0)=(x, 0)$ for all $x$ in $E$. Q.E.D.

Lемма 3.3. For any infinite-dimensional normed linear space $E$, there exists a homeomorphism $i$ of $(E \times(0,1]) \cup(0,0)$ onto $E \times(0,1]$ such that $i(x, 1)=(x, 1)$ for all $x$ in $E$.

Proof. By Lemma 3.2, there exists a homeomorphism $j$ of $E \times[0,1]$ onto $((E \sim\{0\}) \times(0,1]) \cup(E \times\{0\})=((E \sim\{0\}) \times[0,1]) \cup(0,0)$ such that $j(x, 0)=(x, 0)$ for all $x$ in $E$. Define $i_{1}$ of $E \times[0,1]$ onto

$$
((E \sim\{0\}) \times[0,1]) \cup(\{0\} \times\{0,1\})
$$

by $i_{1}(x, t)=j(x, 2 t)$ if $0 \leqq t \leqq 1 / 2 ; i_{1}(x, t)=j(x, 2-2 t)$ if $1 / 2 \leqq t \leqq 1$. It is easy to prove that $i_{1}$ is a homeomorphism and $i_{1}(x, 1)=(x, 1) ; i_{1}(x, 0)=(x, 0)$ for all $x$ in $E$.

Since $E$ is a metric space and $\{0\}$ is conveniently situated in $E$ [11, (2.4)], there exists a homeomorphism $i_{2}$ of $((E \sim\{0\}) \times(0,1]) \cup$ $(\{0\} \times\{0,1\})$ onto $((E \sim\{0\}) \times(0,1]) \cup(\{0\} \times\{1 / 2,1\})$ such that $i_{2}(x, 1)$ $=(x, 1)$ for all $x$ in $E$. Define $i_{3}$ of $((E \sim\{0\}) \times(0,1]) \cup(\{0\} \times\{1 / 2,1\})$ onto $E \times(0,1]$ by $i_{3}(x, t)=i_{1}^{-1}(x, 2 t)$ if $0<t \leqq 1 / 2 ; i_{3}(x, t)=i_{1}^{-1}(x, 2 t-1)$ if $1 / 2 \leqq t \leqq 1$. It can be proved that $i_{3}$ is a homeomorphism such that $i_{3}(x, 1)=(x, 1)$ for all $x$ in $E$. Hence the mapping $i=i_{3} \circ i_{2} \circ i_{1}$ is a homeomorphism of $(E \times(0,1]) \cup(0,0)$ onto $E \times(0,1]$ such that $i(x, 1)=(x, 1)$ for all $x$ in $E$. Q.E.D.

Lemma 3.4. If $F$ is a closed linear subspace of infinite deficiency in a Banach space $E$, then $E$ and $E \sim F$ are homeomorphic. If, in addition, $Q$ is an open half-space in $E$ whose bounding hyperplane $H$ contains $F$ then there exists a homeomorphism $f$ of $Q$ onto $Q \cup F$ such that $f\left(x_{0}+y\right)=y$ for all $y$ in $F$ where $x_{0}$ is in $Q$.

Proof. By Corollary 2.3, there is a homeomorphism $h$ of $E$ onto $(E / F) \times F$ such that $h(x)=(0, x)$ for all $x$ in $F$. Since $E / F$ is an infinite-dimensional Banach space, by Theorem 1.3 , there exists a homeomorphism $g$ of $E / F$ onto $(E / F) \sim\{0\}$. Define

$$
k:(E / F) \times F \rightarrow((E / F) \times F) \sim(\{0\} \times F)
$$

by

$$
k(x, y)=(g(x), y) \quad \text { for all }(x, y) \text { in }(E / F) \times F .
$$

It is clear that $k$ is a homeomorphism of $(E / F) \times F$ onto $((E / F) \times F)$ $\sim(\{0\} \times F)$. The mapping $h^{-1} \circ k \circ h$ is a homeomorphism of $E$ onto $E \sim F$.

By hypothesis, $F$ is contained in the bounding hyperplane $H$ of an open half-space $Q$, hence $H$ is homeomorphic to $(H / F) \times F$. Hence 


$$
\begin{aligned}
& E \approx H \times(-\infty, \infty) \approx(H / F) \times F \times(-\infty, \infty) \approx(H / F) \times(-\infty, \infty) \times F, \\
& Q \approx H \times(0, \infty) \approx(H / F) \times F \times(0, \infty) \approx(H / F) \times(0, \infty) \times F, \\
& Q \cup F \approx((H / F) \times(0, \infty) \times F) \cup(\{0\} \times\{0\} \times F) .
\end{aligned}
$$

To prove $Q$ and $Q \cup F$ are homeomorphic, it suffices to show $(H / F)$ $\times(-\infty, \infty) \times F$ is homeomorphic to

$$
((H / F) \times(0, \infty) \times F) \cup(\{0\} \times\{0\} \times F) .
$$

By Lemma 3.3, there is a homeomorphism $f_{0}$ of $(H / F) \times(0, \infty)$ onto $((H / F) \times(0, \infty)) \cup(0,0)$. Define by

$$
f:(H / F) \times(0, \infty) \times F \rightarrow((H / F) \times(0, \infty) \times F) \cup(\{0\} \times\{0\} \times F)
$$

$$
f(x, t, y)=\left(f_{0}(x, t), y\right) \text { for all }(x, t, y) \text { in }(H / F) \times(0, \infty) \times F .
$$

It is easy to prove that $f$ is a homeomorphism of $(H / F) \times(0, \infty) \times F$ onto $((H / F) \times(0, \infty) \times F) \cup(\{0\} \times\{0\} \times F)$. Q.E.D.

TheOREM 3.5. Let $F$ be a closed linear subspace of infinite deficiency in a Banach space $E$. Let $Q$ be an open half-space in $E$ whose bounding hyperplane $H$ contains $F$. If $K$ is a closed subset in $F$, then $Q$ is homeomorphic to $Q \cup K$ and $E$ is homeomorphic to $E \sim K$.

Proof. By the previous lemma, to prove that $Q$ and $Q \cup K$ are homeomorphic it suffices to show that $Q \cup F$ and $Q \cup K$ are homeomorphic.

For $x$ in $F$, let $\phi(x)=\inf \{\|x-a\| \mid a \in K\}$. Clearly, $\phi$ is a real-valued continuous function on $F$. Define

$$
\begin{aligned}
g:((H / F) \times(0, \infty) \times F) & \cup(\{0\} \times\{0\} \times F) \\
& \rightarrow((H / F) \times(0, \infty) \times F) \cup(\{0\} \times\{0\} \times K)
\end{aligned}
$$

by

$$
g(x, t, y)= \begin{cases}(x, t, y) & \text { if } y \in K, \\ \left(\phi(y) f_{0}^{-1}\left(\frac{x}{\phi(y)}, \frac{t}{\phi(y)}\right), y\right) & \text { if } y \in F \sim K,\end{cases}
$$

where $f_{0}$ is a homeomorphism of $(H / F) \times(0, \infty)$ onto $((H / F) \times(0, \infty))$ $\cup(0,0)$ such that $f_{0}(x, t)=(x, t)$ for $t \geqq 1$ obtained by Lemma 3.3.

To show $g$ is continuous, it suffices to show if $y_{n} \notin K, y_{n} \rightarrow y \in K$, then $g\left(x, t, y_{n}\right) \rightarrow g(x, t, y)$. Since $f_{0}(x, t)=(x, t)$ for $t \geqq 1$, for $n$ sufficiently large, $g\left(x, t, y_{n}\right)=\left(\phi\left(y_{n}\right) f_{0}^{-1}\left(\frac{x}{\phi\left(y_{n}\right)}, \frac{t}{\phi\left(y_{n}\right)}\right), y_{n}\right)=\left(\phi\left(y_{n}\right)\left(\frac{x}{\phi\left(y_{n}\right)}, \frac{t}{\phi\left(y_{n}\right)}\right), y_{n}\right)=\left(x, t, y_{n}\right)$. 
This implies that $g\left(x, t, y_{n}\right) \rightarrow g(x, t, y)$ as $y_{n} \rightarrow y$. Clearly, $g$ is a one-to-one and onto mapping. Define

$$
\begin{aligned}
g_{1}:((H / F) \times(0, \infty) \times F) & \cup(\{0\} \times\{0\} \times K) \\
& \rightarrow((H / F) \times(0, \infty) \times F) \cup(\{0\} \times\{0\} \times F)
\end{aligned}
$$

by

$$
g_{1}(x, t, y)= \begin{cases}(x, t, y) & \text { if } y \in K, \\ \left(\phi(y) f_{0}\left(\frac{x}{\phi(y)}, \frac{t}{\phi(y)}\right), y\right) & \text { if } y \in F \sim K .\end{cases}
$$

Then if $\phi(y)>0$, we have $g \circ g_{1}(x, t, y)=g\left(\phi(y) f_{0}(x / \phi(y), t / \phi(y)), y\right)$ $=\left(\phi(y) f_{0}^{-1}\left(\phi(y) f_{0}^{-1}(x / \phi(y), t / \phi(y)) / \phi(y)\right), y\right)=\left(\phi(y) f_{0}^{-1} \circ f_{0}(x / \phi(y), t / \phi(y)), y\right)$ $=(x, t, y)$. This implies that $g_{1}$ is the inverse of $g$. By similar argument as $g$, it can be proved that $g_{1}$ is continuous. Hence $g$ is a homeomorphism. We have shown that $Q \cup F$ is homeomorphic to $Q \cup K$.

By Lemma 3.4, the mapping $f$ of $(H / F) \times(0, \infty) \times F$ onto

$$
((H / F) \times(0, \infty) \times F) \cup(\{0\} \times\{0\} \times F)
$$

defined by $f(x, t, y)=\left(f_{0}(x, t), y\right)$ for $(x, t, y)$ in $(H / F) \times(0, \infty) \times F$ is a homeomorphism. Hence $g$ of is a homeomorphism of $(H / F) \times(0, \infty) \times F$ onto $((H / F) \times(0, \infty) \times F) \cup(\{0\} \times\{0\} \times K)$ and $g \circ f\left(x_{0}, t_{0}, y\right)=(0,0, y)$ for all $y$ in $K$ where $f_{0}\left(x_{0}, t_{0}\right)=(0,0)$. The restriction of $(g \circ f)^{-1}$ on $(H / F)$ $\times(0, \infty) \times F$ is a homeomorphism of $(H / F) \times(0, \infty) \times F$ onto

$$
((H / F) \times(0, \infty) \times F) \cup\left(\left\{x_{0}\right\} \times\left\{t_{0}\right\} \times K\right) .
$$

This can be used to define a homeomorphism of $E$ onto $E \sim K$. Q.E.D.

REMARK. The restriction of $g$ on

$$
((H / F) \times(0, \infty) \times F) \cup(\{0\} \times\{0\} \times(F \sim K))
$$

is a homeomorphism onto $(H / F) \times(0, \infty) \times F$. Since $K$ is a closed subset of $F, F \sim K$ is an open subset in $F$. This implies that supposing $F$ is a closed linear subspace of infinite deficiency in a Banach space $E$ and $Q$ is an open half-space in $E$ such that the bounding hyperplane of $Q$ contains $F$, then for any open subset $G$ in $F, Q$ is homeomorphic to $Q \cup G$.

REMARK. In case $E$ is an infinite-dimensional Hilbert space, Theorem 3.5 had been proved by Klee [14] implicitly.

Corollary 2.3 is not available in the proof of Theorems $3.1,3.5$ when $E$ is not complete [15]. But if there exists a projection of $E$ onto $F$, then $E=G \times F$ for some closed linear subspace $G$ in $E$. Using the property that $E=G \times F$ in place of Corollary 2.3 we can prove the following results. 
TheOREM 3.6. Let $F$ be a closed linear subspace of finite deficiency in an infinite-dimensional normed linear space $E$. Let $K$ be a closed convex body in $F$.

(a) If the characteristic cone of $K$ is not a linear variety, then $E$ is homeomorphic to $E \sim K$.

(b) If the characteristic cone of $K$ is a linear variety $L$ of infinite deficiency in $F$ and if there exists a continuous projection of $F$ onto $L$, then $E$ is homeomorphic to $E \sim K$.

Theorem 3.7. Let $F$ be a closed linear subspace of infinite deficiency in a normed linear space $E$ such that there exists a continuous projection of $E$ onto $F$. If $K$ is a closed subset of $F$ then $E$ and $E \sim K$ are homeomorphic. If, in addition, $Q$ is an open half-space in $E$ such that the bounding hyperplane of $Q$ contains $F$, then $Q$ and $Q \cup K$ are homeomorphic.

4. Topological equivalence of a Banach space with its closed convex subsets. The problem whether an open half-space $Q$ of an infinite-dimensional Banach space $E$ is homeomorphic to $Q \cup \mathrm{Bd} Q$ is equivalent to whether $E$ is homeomorphic to its unit cell (Theorem 2.4). In this section, we shall consider the problem that if $K$ is a closed convex body of a closed linear subspace of finite deficiency in a Banach space $E$, when is $K$ homeomorphic to $E$ ? We shall first prove two lemmas.

Leмma 4.1. Let $B$ be a Banach space containing a proper closed linear subspace $F$ which is homeomorphic to an infinite-dimensional Hilbert space. If a Banach space $E$ admits a continuous linear mapping $f$ onto $B$, then $E$, $C=\{x \in E \mid\|x\| \leqq 1\}$ and $S=\{x \in E \mid\|x\|=1\}$ are homeomorphic.

Proof. By Theorem 2.2, there exists a homeomorphism $h$ of $E$ onto $P=B \times G, G=f^{-1}(0)$, such that $\|h(x)\|=\|x\|$ for all $x$ in $E$. To prove the theorem, it suffices to show that $P, C_{P}=\{x \in P \mid\|x\| \leqq 1\}$ and $S_{P}$ $=\{x \in P \mid\|x\|=1\}$ are homeomorphic. Let $Y=F \times\{0\} . Y$ is a proper closed linear subspace of $P$ and $Y$ is homeomorphic to an infinite-dimensional Hilbert space $X$. A theorem of Klee [11] assures that $X, X \times(0, \infty)$ and $X \times[0, \infty)$ are homeomorphic. Hence $Y, Y \times(0, \infty)$ and $Y \times[0, \infty)$ are homeomorphic. Since $Y$ is a proper closed linear subspace of $P$, there is a closed hyperplane $H$ in $P$ containing $Y$. Using Corollary 2.3 and the properties of $Y$, we have

$$
\begin{aligned}
P & \approx H \times(0, \infty) \approx(H / Y) \times Y \times(0, \infty) \approx(H / Y) \times Y \times[0, \infty) \\
& \approx H \times[0, \infty) \approx C_{P}, \\
P & \approx H \times(0, \infty) \approx(H / Y) \times Y \times(0, \infty) \\
& \approx(H / Y) \times Y \approx H \approx S_{P} .
\end{aligned}
$$


REMARK 1. This lemma is a consequence of a result of Klee [16]. But the proof is simpler.

REMaRK 2. Klee [13] showed that for every infinite-dimensional normed linear space $E$, the unit cell $C$ of $E$ is homeomorphic to $E \sim$ Int. $C$. Hence the problem whether an infinite-dimensional normed linear space $E$ is homeomorphic to its unit cell $C$ is equivalent to the problem whether $E$ is homeomorphic to $E \sim$ Int.C. Notice that in Theorem 1.3 , we have shown that every infinite-dimensional normed linear space $E$ is homeomorphic to $E \sim C$.

REMarK 3. We have shown that the unit sphere $S$ of an infinitedimensional Banach space $E$ is homeomorphic to a closed hyperplane of $E$. Hence the problem whether every infinite-dimensional Banach space is homeomorphic to its unit sphere is equivalent to the problem whether every infinite-dimensional Banach space is homeomorphic to a closed hyperplane.

Let $F$ be a separable infinite-dimensional closed linear subspace of a Banach space $E$. Let $H$ be a closed hyperplane in $E$ containing $F$. Let $H_{F}$ be a closed hyperplane in $F$. By Corollary $2.3, H \approx\left(H / H_{F}\right) \times H_{F}$ and $E \approx\left(E / H_{F}\right) \times H_{F} \approx\left(H / H_{F}\right) \times(-\infty, \infty) \times H_{F} \approx H / H_{F} \times F$. Hence if $F$ is homeomorphic to $H_{F}$, then $E$ and $H$ are homeomorphic. Now suppose $E$ is a separable infinite-dimensional Banach space, then there exists a continuous linear mapping $f$ of $l_{1}$ onto $E$ [6]. Let $G$ be the kernel of $f$, then $G$ is a closed linear subspace of $l_{1} . E$ is isomorphic to $l_{1} / G$ and the closed hyperplane $H$ of $E$ is isomorphic to $f^{-1}(H) / G$. $f^{-1}(H)$ is a closed hyperplane in $l_{1}$. So the problem is reduced to the following: If $G$ is a closed linear subspace of $l_{1}$ and $H$ is a closed hyperplane in $l_{1}$ containing $G$, does there exist a homeomorphism of $l_{1}$ onto $H$ which maps $G$ onto $G$ ? A recent result of Bessaga and Pe/czyński [3] showed that every infinite-dimensional closed linear subspace of $l_{1}$ is homeomorphic to $l_{1}$.

Lemma 4.2. Let $E$ be a Banach space containing a closed linear subspace $F$ of infinite deficiency which is homeomorphic to an infinite-dimensional Hilbert space. If $L$ is a closed linear subspace of finite deficiency $m$ in $E$, then for each integer $n \geqq 0, E$ and $L \times[0,1]^{n}$ are homeomorphic.

Proof. Since $F$ is a closed linear subspace of infinite deficiency in $E$, there exists a closed linear subspace $M$ of deficiency $m$ in $E$ such that $F$ is contained in $M$. It is easy to define a linear homeomorphism of $E$ onto $E$ which maps $L$ onto $M$. Hence we may assume that $F$ is contained in $L$. Since $F$ is homeomorphic to an infinite-dimensional Hilbert space, $F$ is homeomorphic to $F \times R^{m}$ [11]. Hence we have

$$
E \approx L \times R^{m} \approx(L / F) \times F \times R^{m} \approx(L / F) \times F \approx L .
$$


For $n>0$, to show that $E$ and $L \times[0,1]^{n}$ are homeomorphic, it suffices to show that $E$ and $H \times[0,1]$ are homeomorphic where $H$ is a closed hyperplane in $E$. We may assume that $H$ contains $F$. By Lemma $4.1, H$ and $C_{H}$, the unit cell of $H$, are homeomorphic. Since the unit cell $C$ of $E$ is homeomorphic to $C_{H} \times[0,1]$, we have

$$
E \approx C \approx C_{H} \times[0,1] \approx H \times[0,1] .
$$

Theorem 4.3. Let $E$ be a Banach space containing a closed linear subspace $B$ of infinite deficiency such that $B$ is homeomorphic to an infinite-dimensional Hilbert space. Let $F$ be a closed linear variety of finite deficiency in $E$, and $K$ a closed convex body in $F$.

(a) If the characteristic cone of $K$ is not a linear variety of finite deficiency in $F$, then $E, K$ and $\operatorname{Bd}_{F} K$ are homeomorphic.

(b) If the characteristic cone of $K$ is a linear variety of finite deficiency $n$ in $F$, then $K$ is homeomorphic to $E$ and $\mathrm{Bd}_{F} K$ is homeomorphic to $E \times S_{n-1}$, where $S_{n-1}$ is the $(n-1)$-sphere.

Proof. Without loss of generality, we may assume that $F$ contains $B$.

(a) From Theorems 2.5, 2.6, $K$ is homeomorphic to $C_{F}=\{x \in F \mid\|x\| \leqq 1\}$ and $\mathrm{Bd}_{F} K$ is homeomorphic to $S_{F}=\{x \in F \mid\|x\|=1\}$. Lemma 4.1 implies that $C_{F}, S_{F}$ and $F$ are homeomorphic. Hence $K, \mathrm{Bd}_{F} K$ and $F$ are homeomorphic. But $F$ is homeomorphic to $E$ by Lemma 4.2 . This shows that $K$ and $\mathrm{Bd}_{F} K$ are homeomorphic to $E$.

(b) By Theorem 2.7, $K$ is homeomorphic to $L \times C_{n}$ and $\mathrm{Bd}_{F} K$ is homeomorphic to $L \times S_{n-1}$. Lemma 4.2 assures that $F, L$ and $L \times[0,1]^{n}$ are homeomorphic. Hence $K$ is homeomorphic to $F$ and $\mathrm{Bd}_{F} K$ is homeomorphic to $F \times S_{n-1}$. But $F$ is homeomorphic to $E$. The proof of the theorem is completed. Q.E.D.

REMARK. Lemma 4.1 and Theorem 4.3 should be compared with the following result of Corson and Klee [4]: Suppose the normed linear space $E$ admits (for each finite $n$ ) a closed linear subspace of deficiency $n$ which is homeomorphic with its own unit cell. Then $E$ is homeomorphic with all its closed convex bodies.

REMARK 2. Every infinite-dimensional Banach space clearly contains a separable infinite-dimensional proper closed linear subspace. If Banach's conjecture that all separable infinite-dimensional Banach spaces are homeomorphic is true, then every infinite-dimensional Banach space would contain a proper closed linear subspace homeomorphic to $l_{2}$. Hence if Banach's conjecture is true, Theorem 4.3 would imply that if $K$ is a closed convex body in a closed linear subspace of finite deficiency in an infinite-dimensional Banach space $E$, then $K$ is homeomorphic to $E$ and $\operatorname{Bd}_{F} K$ is homeomorphic to $E$ or $E \times S_{n}$ for some integer $n \geqq 0$. 
REMARK 3. If $E$ is an infinite-dimensional Hilbert space, then every infinite-dimensional closed linear subspace $F$ of $E$ is isomorphic to $E$. Let $K$ be a closed convex body in $F$. By Theorem $4.3, K$ is homeomorphic to $F$, hence to $E$. Klee [12] had proved that every locally compact closed convex subset of an infinite-dimensional normed linear space is homeomorphic either to $[0,1]^{m} \times(0,1)^{n}$ or $[0,1]^{m} \times[0,1)$, where $m, n$ are cardinal numbers, $0 \leqq m \leqq \boldsymbol{\aleph}_{0}, 0 \leqq n<\boldsymbol{\aleph}_{0}$. Hence it is natural to ask whether in an infinite-dimensional Hilbert space $E$, there exists a closed convex but not locally compact subset $K$, which is not contained in any proper closed linear subspace of $E$ and every point of $K$ is a boundary point. The answer is positive. Let $K=\left\{x \in l_{2} \mid x=\left(x_{1}, x_{2}, \cdots\right), x_{i} \geqq 0\right.$ for all $\left.i=1,2, \cdots\right\}$. It is clear that $K$ is closed convex and is not contained in any proper closed linear subspace of $l_{2}$. Klee [10] proved that every point of $K$ is a boundary point. It can be proved that every neighborhood of $(0,0, \ldots)$ in $K$ is not compact. The topological classification of all closed convex subsets of an infinite-dimensional Hilbert space is far from complete.

The hypothesis of Theorem 4.3 lead us to consider the problem: When is an infinite-dimensional separable Banach space homeomorphic to $l_{2}$ ? Each of the following separable infinite-dimensional Banach spaces $E$ is homeomorphic to $l_{2}$.

(a) $E$ is a $w^{*}$-closed linear subspace of the dual space of a normed linear space.

(b) $E$ is $\left(c_{0}\right)$, the space of all sequences converging to 0 with supremum norm.

(c) $E$ admits an unconditional basis.

(d) $E$ is a closed linear subspace of $L(X, \mu)$ of all real-valued $\mu$-absolutely summable functions on a compact metric space $X$ and $\mu$ is a Borel measure on $X$.

5. Periodic homeomorphisms with preassigned set of fixed points. This section is devoted to Problem II. The main results are Theorems 5.2, 5.3.

Lemma 5.1. Let $E$ be a normed linear space. For every positive integer $n$, there exists a homeomorphism of period $2 n$ of $E$ onto $E$ with 0 as the only fixed point.

Proof. Let $F$ be a two-dimensional closed linear subspace of $E$. Then there exist a closed linear subspace $G$ of $E$ and a homeomorphism $h_{1}$ of $E$ onto $G \times F$ such that $h_{1}(0)=(0,0)$ and $h_{1}(x)=(0, x)$ for all $x$ in $F$. For each positive integer $n$, there exists a homeomorphism of period $n$ of the Euclidean plane $R_{2}$ onto itself with 0 as the only fixed point. Since $F$ is topologically equivalent to $R_{2}$, there exists a homeomorphism $k$ of period $n$ of $F$ onto $F$ with 0 as the only fixed point. Define 


$$
h_{2}: G \times F \rightarrow G \times F
$$

by

$$
h_{2}(x, y)=(-x, k(y)) \text { for }(x, v) \text { in } G \times F .
$$

Then $h_{2}$ is a homeomorphism of period $2 n$ of $G \times F$ onto $G \times F$ with $(0,0)$ as the only fixed point. Hence $h_{1}^{-1} \circ h_{2} \circ h_{1}$ is a homeomorphism of period $2 n$ of $E$ onto $E$ with 0 as the only fixed point. Q.E.D.

Theorem 5.2. Let $F$ be an infinite-dimensional closed linear subspace of a Banach space $E$. If the characteristic cone of a closed convex body $K$ of $F$ is not a linear variety of finite deficiency in $F$ then for each positive integer $n$, there exists a homeomorphism of period $2 n$ of $E$ onto $E$ with $\operatorname{Bd}_{F} K$ as its set of fixed points. In case $E$ is an infinite-dimensional Hilbert space, then for each integer $n>1$, there exists a homeomorphism of period $n$ of $E$ onto $E$ with $K$ as its set of fixed points.

Proof. Let $H$ be a closed hyperplane in $E$ containing $F$ (in case $F=E$, let $H$ be the space $E$ ). Let $H_{F}$ be a closed hyperplane in $F . H_{F}$ is a closed linear subspace of $H$. Corollary 2.3 implies that there is a homeomorphism $h_{1}$ of $E$ onto $\left(H / H_{F}\right) \times H_{F} \times(-\infty, \infty)$. By Theorems $2.5,2.6$, there is a homeomorphism $h_{2}$ of $H_{F} \times(-\infty, \infty)$ onto $H_{F} \times(-\infty, \infty)$ such that $h_{2}(K)$ $=H_{F} \times[0, \infty), h_{2}\left(\mathrm{Bd}_{F} K\right)=H_{F} \times\{0\}$. Define $h_{3}$ of $\left(H / H_{F}\right) \times H_{F} \times(-\infty, \infty)$ onto itself by $h_{3}(x, y, t)=(r(x), y,-t)$ for all $(x, y, t)$ in $\left(H / H_{F}\right) \times H_{F}$ $\times(-\infty, \infty)$, where $r$ is a homeomorphism of period $2 n$ of $H / H_{F}$ onto itself with 0 as the only fixed point. $h_{3}$ is a homeomorphism of period $2 n$ of $\left(H / H_{F}\right) \times H_{F} \times(-\infty, \infty)$ onto itself with $\{0\} \times H_{F} \times\{0\}$ as its set of fixed points. Hence $h_{1}^{-1} \circ\left(1 \times h_{2}\right)^{-1} \circ h_{3} \circ\left(1 \times h_{2}\right) \circ h_{1}$ is a homeomorphism of period $2 n$ of $E$ onto $E$ with $\mathrm{Bd}_{F} K$ as its set of fixed points.

Suppose $E$ is an infinite-dimensional Hilbert space. Since $F$ is an infinitedimensional closed linear subspace of $E, F$ is isomorphic to $E$. By a theorem of Klee $[11$, p. 33], for each integer $n>1$, there exists a homeomorphism $k_{1}$ of period $n$ of $F$ onto $F$ with $\left(F \sim \operatorname{Int} . C_{F}\right)=\{x \in F \mid\|x\| \geqq 1\}$ as its set of fixed points. On the other hand, there is a homeomorphism $k_{2}$ of $F$ onto $F$ such that $k_{2}\left(C_{F}\right)=F \sim$ Int. $C_{F}[13]$. By Theorems 2.5, 2.6, there is a homeomorphism $k_{3}$ of $F$ onto $F$ such that $k_{3}(K)=C_{F}$. Hence $k=k_{3}^{-1} \circ k_{2}^{-1}$ $\circ k_{1} \circ k_{2} \circ k_{3}$ is a homeomorphism of period $n$ of $F$ onto $F$ with $K$ as its set of fixed points. It is clear that $k$ can be used to define a homeomorphism of period $n$ of $E$ onto $E$ with $K$ as its set of fixed points. Q.E.D.

Theorem 5.3. Let $F$ be a closed linear subspace of infinite deficiency in a Banach space $E$. If $K$ is a closed subset in $F$ then, for each positive integer $n$, there exists a homeomorphism of period $2 n$ of $E$ onto $E$ with $K$ as its set of fixed points. In case $E$ is an infinite-dimensional Hilbert space, for each integer 
$n>1$, there exists a homeomorphism of period $n$ of $E$ onto $E$ with $K$ as its set of fixed points.

Proof. Let $Q$ be an open half-space in $E$ such that the bounding hyperplane $H$ of $Q$ contains $F$. By Theorem 3.5, there is a homeomorphism $h_{2}$ of $Q$ onto $Q \cup K$ such that $h_{2}\left(x_{0}+y\right)=y$ for all $y$ in $K$ where $x_{0} \in Q$. Let $h_{1}$ be a homeomorphism of $E$ onto $Q$ such that $h_{1}(y)=x_{0}+y$ for all $y$ in $H$. Corollary 2.3 asserts that there is a homeomorphism $h_{3}$ of $Q \cup K$ onto $((H / F) \times(0, \infty) \times F) \cup(\{0\} \times\{0\} \times K)$. Since $H / F$ is an infinitedimensional Banach space, by Lemma 3.2, there is a homeomorphism $j$ of $(H / F) \times[0, \infty)$ onto $(((H / F) \sim\{0\}) \times(0, \infty)) \cup((H / F) \times\{0\})$ such that $j(x, 0)=(x, 0)$ for all $x$ in $H / F$. Define

$h_{4}:((H / F) \times(0, \infty) \times F) \cup(\{0\} \times\{0\} \times K)$

$$
\rightarrow(((H / F) \sim\{0\}) \times(0, \infty) \times F) \cup(\{0\} \times\{0\} \times K)
$$

by $h_{4}(x, t, y)=(j(x, t), y)$ for $x \in H / F, t \in[0, \infty), y \in F$. It can be proved that $h_{4}$ is a homeomorphism of $((H / F) \times(0, \infty) \times F) \cup(\{0\} \times\{0\} \times K)$ onto $(((H / F) \sim\{0\}) \times(0, \infty) \times F) \cup(\{0\} \times\{0\} \times K)$ such that $h_{4}(0,0, y)$ $=(0,0, y)$ for all $y$ in $K$. Define $h_{5}$ of $(((H / F) \sim\{0\}) \times(0, \infty) \times F)$ $\cup(\{0\} \times\{0\} \times K)$ onto itself by $h_{5}(x, t, y)=(r(x), t, y)$ where $r$ is a homeomorphism of period $2 n$ of $H / F$ onto itself with 0 as the only fixed point obtained by Lemma 5.1. It is clear that $h_{5}$ is a homeomorphism of period $2 n$ with $\{0\} \times\{0\} \times K$ as its fixed-point set. Then the mapping $h_{1}^{-1} \circ h_{2}^{-1} \circ h_{3}^{-1}$ $\circ h_{4}^{-1} \circ h_{5} \circ h_{4} \circ h_{3} \circ h_{2} \circ h_{1}$ is a homeomorphism of period $2 n$ of $E$ onto $E$ with $K$ as its set of fixed points.

If $E$ is an infinite-dimensional Hilbert space, then $H / F$ is isomorphic to $E$. Hence for each integer $n>1$, there exists a rotation $r$ of angle $2 \pi / n$ of $H / F$ onto $H / F$ with 0 as the only fixed point (see [1] or [11]). Define $h_{5}^{\prime}$ of $(((H / F) \sim\{0\}) \times(0, \infty) \times F) \cup(\{0\} \times\{0\} \times K)$ onto itself by $h_{5}^{\prime}(x, t, y)=(r(x), t, y)$. Then $h_{1}^{-1} \circ h_{2}^{-1} \circ h_{3}^{-1} \circ h_{4}^{-1} \circ h_{5}^{\prime} \circ h_{4} \circ h_{3} \circ h_{2} \circ h_{1}$ is a homeomorphism of period $n$ of $E$ onto $E$ with $K$ as its fixed-point set. Q.E.D.

REMARK. In the case when $E$ is an infinite-dimensional Hilbert space, Theorem 5.3 had been proved implicitly by Klee [14].

Corollary 2.3 is not available in the proof of Theorems 5.2, 5.3, when $E$ is not complete [15]. But if there exists a continuous projection of $E$ onto $F$, then $E=G \times F$ for some closed linear subspace $G$ in $E$. Using the property that $E=G \times F$ in place of Corollary 2.3 we can prove the following results.

THEOREM 5.4. Let $F$ be a closed linear subspace of finite deficiency in an infinite-dimensional normed linear space $E$. Let $K$ be a closed convex body in $F$. 
(a) If the characteristic cone of $K$ is not a linear variety then for each positive integer $n$, there exists a homeomorphism of period $2 n$ of $E$ onto $E$ with $\mathrm{Bd}_{F} K$ as its fixed-point set.

(b) If the characteristic cone of $K$ is a linear variety $L$ of infinite deficiency in $F$ and if there exists a continuous projection of $F$ onto $L$, then for each positive integer $n$, there exists a homeomorphism of period $2 n$ of $E$ onto $E$ with $\mathrm{Bd}_{F} K$ as its set of fixed points.

Theorem 5.5. Let $F$ be a closed linear subspace of infinite deficiency in a normed linear space $E$ such that there exists a continuous projection of $E$ onto $F$. For every closed subset $K$ of $F$ and for each positive integer $n$, there exists a homeomorphism of period $2 n$ of $E$ onto $E$ with $K$ as its set of fixed points.

Theorem 5.3 leads us to consider the following problem: Given a compact subset $K$ of an infinite-dimensional topological vector space $E$, does there exist a homeomorphism $i$ of $E$ onto $E$ such that $i(K)$ is contained in a closed linear subspace of infinite deficiency in $E$ ? Unfortunately, we only have the following partial result due to Klee [12]. For any given nonnegative integer $n$ and compact subset $K$ of an infinite-dimensional Banach space $E$, there exist closed linear subspaces $L_{1}, L_{2}$ of $E, E=L_{1} \oplus L_{2}$, $\operatorname{dim} L_{2}=n$, and an isotopy $f$ on $E$ such that $f_{1}$ maps $K$ linearly into $L_{1}$ and $f_{0}$ is the identity mapping on $E$. It can be proved that the result is true even when $E$ is an infinite-dimensional complete metrizable locally convex topological vector space.

\section{Bibliography}

1. S. Banach, Théories des opérations linéaires, Monografje Matematyczne, Warsaw, 1932.

2. R. G. Bartle and L. M. Graves, Mappings between function spaces, Trans. Amer. Math. Soc. 72 (1952), 400-413.

3. C. Bessaga and A. Pelczyński, Some remarks on homeomorphisms of Banach spaces, Bull. Akad. Polon. Sci. 8 (1960), 757-761.

4. H. H. Corson and V. L. Klee, Topological classification of convex sets, Proc. Sympos. Pure Math. Vol. 7, pp. 37-51, Amer. Math. Soc., Providence, R. I., 1963.

5. J. Dugundji, An extension of Tietze's theorem, Pacific J. Math. 1 (1951), 353-367.

6. N. Dunford and J. Schwartz, Linear operators, Vol. I, Interscience, New York, 1958.

7. R. C. James, Bases and reflexivity of Banach spaces, Ann. of Math. (2) 52 (1950), 518-527.

8. M. I. Kadeč, On homeomorphism of certain Banach spaces, Dokl. Akad. Nauk SSSR 92 (1953), 465-468.

9. __ On weak and norm convergence, Dokl. Akad. Nauk SSSR 122 (1958), 13-16.

10. V. L. Klee, Jr., The supporting property of a convex set in a linear normed space, Duke Math. J. 15 (1948), 767-772.

11. Convex bodies and periodic homeomorphisms in Hilbert space, Trans. Amer.

Math. Soc. 74 (1953), 10-43.

12. Some topological properties of convex sets, Trans. Amer. Math. Soc. 78 (1955), $30-45$.

13. Soc. 7 (1956), 673-674. 
14. - Fixed-point sets of periodic homeomorphisms of Hilbert space, Ann. of Math. (2) 64 (1956), 393-395.

15.

16. , Topological equivalence of a Banach space with its unit cell, Bull. Amer. Math. Soc. 67 (1961), 286-290.

17. E. Michael, Selected selection theorems, Amer. Math. Monthly 63 (1956), 233-238.

18. , Continuous selections. I, Ann. of Math. (2) 63 (1956), 361-382.

19. P. A. Smith, Fixed point theorems for periodic transformations, Amer. J. Math. 63 (1941), 1-8.

20. J. J. Stoker, Unbounded convex point sets, Amer. J. Math. 62 (1940), 165-179.

NORTHWESTERN UNIVERSITY,

Evanston, Illinois

State UnIVERSITY OF Iowa, Iowa CrTy, Iowa 